\title{
SOME RECENT RESULTS \\ IN HIGH PRESSURE ARC DISCHARGE RESEARCH
}

\author{
U. H. BAUDER \\ Electrophysical Institute \\ Technical University, Munich, Germany
}

\begin{abstract}
1. Introduction. - There exist at least two ways of presenting a review topic such as the present topic. One approach, taken most frequently is particularly applicable to well defined and confined research areas. In this case a total overview of the present status of research can be given. For broad research areas consisting of overlapping disciplines such as the field of high density plasma research, it is impossible to take this approach within the boundary conditions of a journal publication. The alternative is to restrict the presentation to a few selected topics which are presently under active study. I have chosen to follow this route by giving an overview of some work being done presently in Munich. The discussion will touch on the fields of plasma diagnostics and report on some recent transport property work.
\end{abstract}

The research in the discussed areas has been stimulated by applications of high pressure arc discharges such as high power circuit breakers and arc heaters (plasmatrons) for atmospheric entry simulation or for plasma chemistry. Following the generally accepted nomenclature, high pressure arc discharges cover the pressure range from atmospheric pressure to several hundreds of atmospheres, the temperatures found in steady state discharges extend to approximately $30000 \mathrm{~K}$. This combination of pressure/temperature values provides for the condition of local thermodynamic equilibrium to hold in general in such discharges, exceptions being small areas with exceedingly large temperature gradients and discharges in a few selected gases, such as helium. On the other hand, radiation reabsorption may play an important role in the energy balance of such discharges. Plasma diagnostical applications as well as transport property work directed toward the determination of the radiative source strength and the thermal conductivity of plasmas may be affected by absorption.

2. Diagnostics of high pressure discharges. - For the development of plasma diagnostical techniques per se as well as for the generation of atomic data, useful for spectroscopic and interferometric applications, the cascade arc as described by Maecker [1] provides an excellent tool. Originally this type of arc had been designed for transport property work. In this application, the absence of convective energy losses of the column, together with the perfect cylindrical symmetry are the desirable features. In diagnostical applications, the symmetry of this discharge type is equally important; in addition however, it provides special advantages for work in the Vacuum-UV (VUV)-region. Figure 1 gives a schematic of the

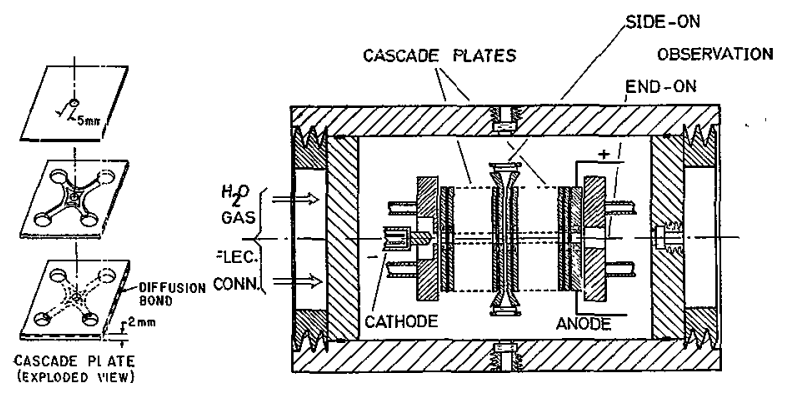

Fig. 1. - The high pressure cascade arc. Left side : exploded view of an individual cascade plate.

cascade arc as used in high pressure applications. The cascade channel wall is segmented, it confines and stabilizes the arc column. The cascade consists of individually cooled copper plates (Fig. 1 also gives an exploded view of a single cascade plate) sandwiched together with insulating spacers separating the copper plates. The special advantage of this arc type in spectroscopic applications in the VUV-wavelength region results from the possibility of introducing different types of gas into different sections of the cascade tube. By this technique it is possible, to create short transition zones between plasma regions containing different gases. Thus, in end-on observation through a hollow electrode, even the resonance radiation which originates in the central cascade section can leave the cascade without significant absorption in the outer cold layers of gas of identical or of different chemical species. Instead of using a gas blanket in the end region of the arc, it is equally possible, to evacuate this region by differential pumping. Boldt [2] used the blanket technique to produce a VUV radiation standard consisting of optically thick blackbody limited resonance lines. 
More recently Behringer, Gieres and Ott [3] approached the realisation of a radiation standard in the VUV-region differently by proposing for this purpose the use of the continuous radiation emitted from a wall stabilized hydrogen arc under optically thin conditions. In this application, the high power density capability of the cascade arc plays the important role. By operating the arc at high arc currents, gas temperatures exceeding $20000 \mathrm{~K}$ may be reached in the axis of the hydrogen column. On the other hand, the so called norm temperature of the hydrogen continuous radiation in the wavelength range between $150 \mathrm{~nm}$ and $250 \mathrm{~nm}$ lies below $20000 \mathrm{~K}$ (Fig. 2). The norm temperature is the temperature at which the emission coefficient of a particular radiative mechanism exhibits a maximum for the given pressure level (FowlerMilne maximum). This temperature maximum is due to the counteracting effects of increasing degree of ionisation with rising temperature and decreasing total number density. In arc discharges with offaxis maxima of emission coefficients $\left(\varepsilon_{v}\right)$ the centerline temperature is higher than the norm temperature. Following the method described by Larenz [4] this $\varepsilon_{v}$-maximum which is exactly known from theory, may be used for calibration purposes for the rest of the radiation leaving the column. By using a hydrogen cascade arc of $2 \mathrm{~mm}$ diameter and $5.3 \mathrm{~cm}$ length at arc currents between $60 \mathrm{~A}$ and $100 \mathrm{~A}$ a norm temperature primary radiation standard for the VUV region was established. The particular merits of this standard are its insensitivity to misalignments (the major part of the arc diameter exhibits nearly identical emission coefficients) and to uncertainties in the arc current.

In a recent publication [5] this continuum radiation emitted under conditions of small optical depth from a hydrogen cascade arc was compared to synchroton radiation which also serves as radiation

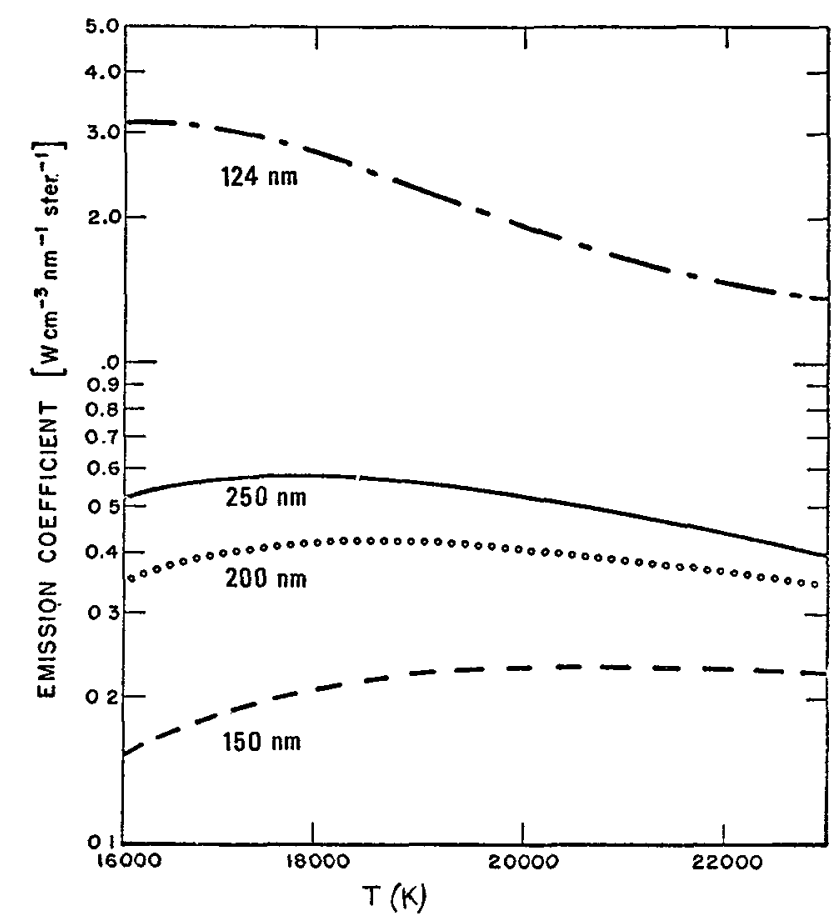

Fig. 2. - Continuous radiation of hydrogen as function of temperature [3].

standard. For the wavelength range extending from $175-340 \mathrm{~nm}$ it results excellent agreement. The ratio of the two spectral radiances has a mean value of 1.03 and thereby remains well within the uncertainties due to calibration.

By using the hydrogen continuum radiation standard, new sets of argon atomic data have been generated from cascade arc measurements [6, 7]. In this experiment, it was possible to use the same arc chamber for the argon runs (measurement of line and continuum intensities) and for the runs with hydrogen (absolute calibration of the total optical system).

TABLE I

Transition probabilities for UV ArII lines [6]

\begin{tabular}{|c|c|c|c|c|c|}
\hline \multirow[b]{3}{*}{$\lambda / \mathrm{nm}$} & \multirow[b]{3}{*}{ Transition } & \multicolumn{4}{|c|}{$A_{m n} / 10^{8} \mathrm{~s}^{-1}$} \\
\hline & & \multicolumn{2}{|c|}{ Ref. [6] } & \multirow[b]{2}{*}{ Ref. [9] } & \multirow[b]{2}{*}{ Ref. [8] } \\
\hline & & $19500 \mathrm{~K}$ & $21500 \mathrm{~K}$ & & \\
\hline 30001 & $\left.3 d^{\prime 2} F_{-\left({ }^{3} \mathrm{P}\right.}\right) 4 f\{3\}^{0}$ & \multirow{2}{*}{\multicolumn{2}{|c|}{0.041}} & - & \\
\hline 300.01 & $3 \mathrm{~d}^{\prime 2} \mathrm{~F}_{5 / 2}-\left({ }^{3} \mathrm{P}_{0}\right)$ 4f $\{3\}_{7 / 2}^{0}$ & & & & \\
\hline 300.04 & $4 p^{2} D_{3 / 2}^{\delta^{\prime}}-4 d^{2} D_{3 / 2}$ & \multicolumn{2}{|c|}{0.283} & 1.5 & 0.273 \\
\hline 318.10 & $4 p^{4} P_{5 / 2}^{0}-5 d^{4} P_{3 / 2}^{5 / 2}$ & \multicolumn{2}{|c|}{0.372} & 0.63 & 0.387 \\
\hline 292.46 & $3 \mathrm{~d}^{\prime}{ }^{2} \mathrm{P}_{3 / 2}-\left({ }^{1} \mathrm{D}\right) 4 \mathrm{f}\{1\}_{3 / 2}^{0}$ & 0.458 & 0.474 & & \\
\hline 301.45 & $4 \mathrm{p}^{2} \mathrm{D}_{3 / 2}^{0^{\prime}}-4 \mathrm{~d}^{2} \mathrm{D}_{5 / 2}$ & 0.043 & 0.044 & & 0.032 \\
\hline 303.35 & $4 \mathrm{~s}^{2} \mathbf{P}_{1 / 2}-4 \mathrm{p}^{\prime 2} \mathbf{P}_{3 / 2}^{0}$ & 0.088 & 0.088 & & \\
\hline 316.97 & $4 p^{4} P_{3 / 2}^{0}-4 d{ }^{4} P_{5 / 2}$ & 0.423 & 0.447 & 0.82 & 0.565 \\
\hline 322.60 & $4 p^{4} P_{3 / 2}^{0}-4 d^{4} F_{3 / 2}$ & 0.017 & 0.018 & 0.046 & \\
\hline 324.37 & $4 p^{4} P_{3 / 2}^{0}-4 d^{4} P_{1 / 2}$ & 0.905 & 0.969 & 2.0 & 0.541 \\
\hline 324.98 & $4 p^{4} P_{1 / 2}^{0^{\prime}}-4 d^{4} P_{3 / 2}$ & 0.623 & 0.616 & 1.0 & 1.06 \\
\hline 326.36 & $4 p^{4} P_{1 / 2}^{1 / 2}-4 d^{4} F_{3 / 2}^{3 / 2}$ & 0.131 & 0.123 & 0.35 & \\
\hline 328.17 & $4 \mathrm{p}^{4} \mathrm{P}_{1 / 2}^{0^{\prime}}-4 \mathrm{~d}^{4} \mathrm{P}_{1 / 2}$ & 0.404 & 0.382 & 0.73 & 0.215 \\
\hline
\end{tabular}


Additional confirmation for the absolute intensity values was obtained by operating the arc at a current of $180 \mathrm{~A}$ which leads to a centerline temperature of $26500 \mathrm{~K}$, corresponding to the norm temperature of ArII lines. The transition probabilities of several ArII lines in the wavelength region between $300-330 \mathrm{~nm}$ were then deduced from the measured total intensities of these lines. The values are reproduced in table $I$. For comparison theoretical results obtained by Griem [8] and $A_{m n}$ values, tabulated by Wiese [9] are also listed.

The results of the argon continuum measurements in the VUV range are shown in figure 3 for three different arc currents. A comparison with theoretical emission coefficients computed by using Schlüters [10] approach for the determination of the $\xi$-factor demonstrates excellent agreement for low temperatures $(T=16900 \mathrm{~K})$. At high temperatures $(T=21500 \mathrm{~K})$ the deviation becomes substantial in the range 140$230 \mathrm{~nm}$, c.e. close to the recombination edges for $\mathrm{A}(\mathrm{III}) \rightarrow \mathrm{A}(\mathrm{II})$. Behringer and Thoma assume that the excess radiation found in this region may be due

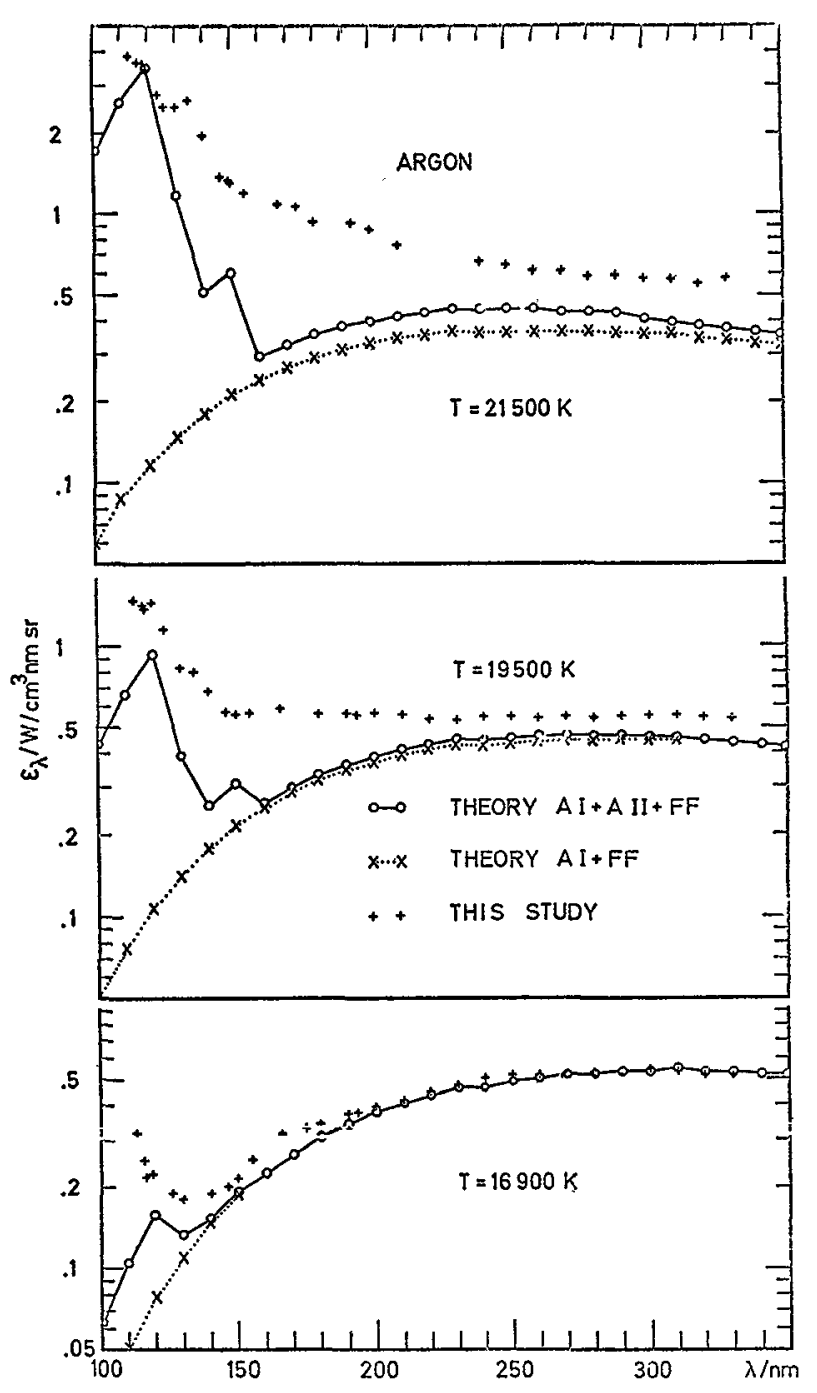

FIG. 3. - Argon continuous radiation at different temperatures [7]. to high lying $\operatorname{Ar}(\mathrm{II})$ levels which are strongly broadened and thereby lead to a pseudo-continuum.

3. Transport properties of nitrogen and air. - The cascade arc technique for the determination of transport properties has proven its usefulness during the past decade [11]. The properties which can be determined as function of temperature and pressure from measurements on wall stabilized arcs are : $\sigma$ (electrical conductivity), $k$ (thermal conductivity), $e$ (radiation source strength), $\eta$ (viscosity). In cylindrical arc columns with negligible radiation reabsorption, the energy equation for an element of volume is given by the well publicized Elenbaas-Heller equation :

$$
\sigma E^{2}+\frac{1}{r} \frac{\mathrm{d}}{\mathrm{d} r}\left(r k \frac{\mathrm{d} T}{\mathrm{~d} r}\right)-e=0
$$

where the first term corresponds to the energy input by Ohmic heating ( $E=$ electric fieldstrength), while the second and third terms of eq. (1) are loss terms relating to thermal conduction and optically thin radiation respectively.

Unfortunately, arc discharges operating in gases which are useful in applications, such as $\mathrm{Ar}, \mathrm{N}_{2}$, air and with temperatures exceeding $10000 \mathrm{~K}$ usually are influenced by radiation reabsorption. In this case, a detailed study of the radiative processes and the corresponding photon path length $\lambda_{\text {ph }}$ has to be carried out to insure the validity of eq. (1) in cases where the mean value $\lambda_{\mathrm{ph}}$ is large compared to the geometrical dimensions of the discharge. This condition is practically never fulfilled for resonance radiation leading to the ground state of the particular system. However, as Kopainsky [12] has shown for argon discharges, it may be possible to treat this resonance radiation as a photon diffusion process, leading to an additional contribution to the thermal conductivity. In this case, the $k(T)$ determined from the experiment will exceed the theoretical values which contain only the contact conductivities of the different species together with the transport of excitation and ionisation energies. Regardless of the absorption condition, the electrical conductivity can always be obtained directly from the solution of the integral eq. (2) relating arc current (I) arc radius $R, E$ and $\sigma$ :

$$
I=2 \pi E R^{2} \int_{0}^{1} \sigma \rho \mathrm{d} \rho
$$

where $\rho=r / R$.

Eq. (2) can be solved by numerical methods to yield $\sigma(\rho)$ from measured data. The function $\sigma(\rho)$ being known from this procedure, $T(\rho)$ data of the discharge may then be used of generate experimental $\sigma(T)$ values. Figure 4 gives an example of such a $\sigma(T)$ determination in an air plasma [13]. The comparison with results of other experiments and with theory demonstrates satisfactory agreement within the error 


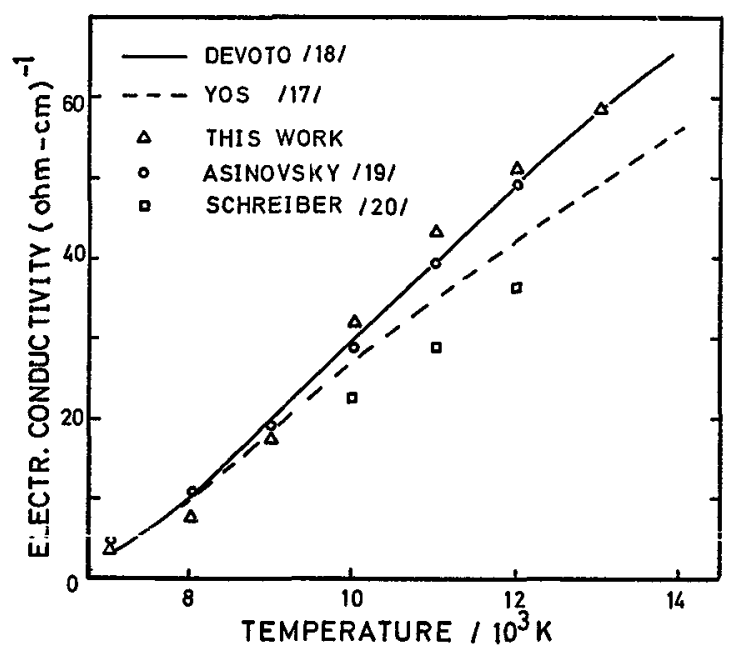

FIG. 4. - Electrical conductivity of air at $p=1$ atm. [13].

limits of the method. Thus, the electrical but not the radiative energy input to the volume element are known in eq. (1). The problem associated with radiation reabsorption corresponding to photon path lengths which are of the order of magnitude of the plasma dimensions, arises from the fact that in this case the radiative term of eq. (1) has to be written as a balance term between energy emitted $e$ and energy absorbed $a$ :

$$
u=e-a .
$$

While $e=e(T)$ is a function of temperature only, $a$ contains the absorption coefficient and therefore depends on the local temperature. However, it also is influenced by the $T(r)$ profile and by the arc geometry.

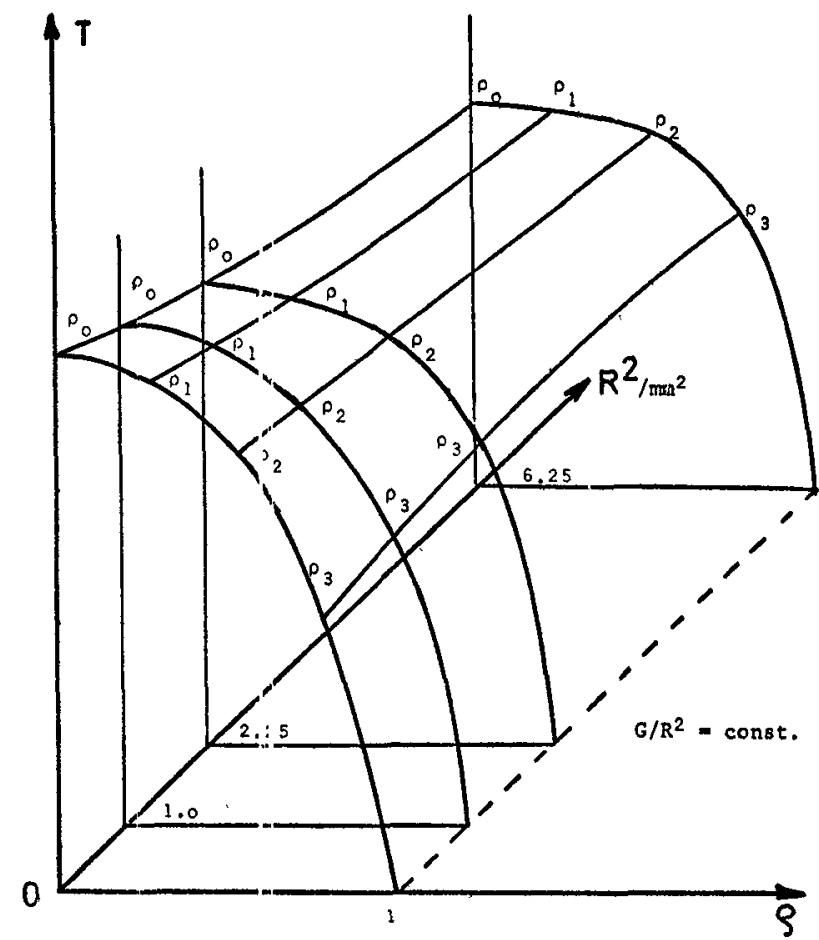

Due to the lack of the relevant atomic data, exact computations of the different radiative processes are impossible in complex spectra, such as nitrogen or air. For the determination of the thermal conductivity and the radiative source strength from cascade arc experiments with non-negligible absorption there exists a special technique described first by Asinovsky [14] and Ernst [15] avoiding such radiative computations. The basic idea consists of decreasing the radiative contribution to the energy balance by investigating this balance for an arc with vanishing diameter. An extrapolation procedure is used, several sets of data obtained in arcs of different diameters are required. Defining the conductance of the arc channel as

$$
G=I / E
$$

this conductance is known from the measured electrical arc characteristic $E(I)$. Ernst noted that arcs of different radii $(R)$ but with identical normalized conductances

$$
\frac{G}{R^{2}}=\frac{I}{E R^{2}}=2 \pi \int_{0}^{1} \sigma \rho \mathrm{d} \rho=\pi \int_{0}^{1} \sigma \mathrm{d}\left(\rho^{2}\right)
$$

exhibit temperature profiles $T(\rho)$ which cover very closely the same area under the curves. Considering eq. (5) this similarity becomes plausible by noting that $G / R^{2}=$ const. signifies

$$
\int_{0}^{1} \sigma \mathrm{d} \rho^{2}=\text { const. }
$$

and that in the temperature range of interest, $\sigma$ increases approximately proportional to the temperature.

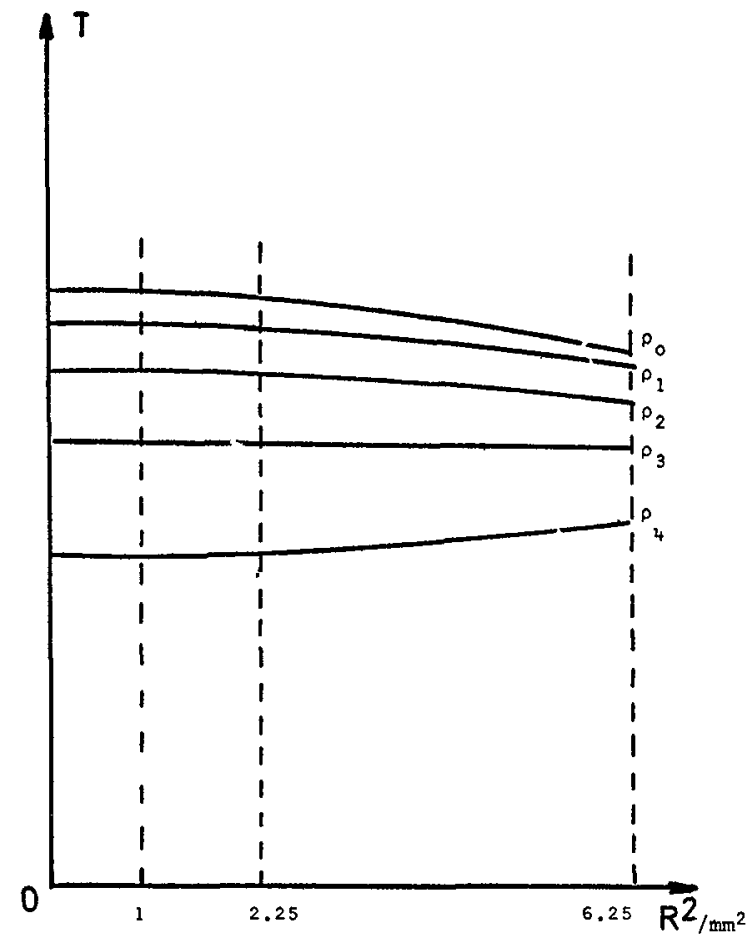

FIG. 5. - Extrapolation of temperature profiles in an arc with vanishing diameter. $T\left(\rho \rightarrow 0, G / R^{2}=\right.$ const.) [15]. 
Assuming that a sufficient dense series of $T\left(\rho, G / R^{2}\right)$ profiles has been measured in arcs of different radii, an extrapolation for a selected $G / R^{2}=$ const. value towards a profile $T\left(\rho \rightarrow 0, G / R^{2}=\right.$ const.) may .be performed. Figure 5 gives a schematic of the type of data extrapolation which is to be performed for different normalized conductivities. Integrating eq. (1) formally to a radius $\rho$ yields :

$$
\begin{aligned}
& 2 \pi(E R)^{2} \int_{0}^{\rho} \sigma \rho^{\prime} \mathrm{d} \rho^{\prime}+ \\
& \quad+2 \pi k \rho \frac{\mathrm{d} T}{\mathrm{~d} \rho}-2 \pi R^{2} \int_{0}^{\rho} e \rho^{\prime} \mathrm{d} \rho^{\prime}=0
\end{aligned}
$$

The energy balance (7) may be solved for the thermal conductivity $k$ :

$$
k=-\frac{(E R)^{2} \int_{0}^{\rho} \sigma \rho^{\prime} \mathrm{d} \rho^{\prime}}{\rho \frac{\mathrm{d} T}{\mathrm{~d} \rho}}+\frac{R^{2} \int_{0}^{\rho} e \rho^{\prime} \mathrm{d} \rho^{\prime}}{\rho \frac{\mathrm{d} T}{\mathrm{~d} \rho}} .
$$

Having $\sigma(T)$ determined from eq. (2), the following procedure may be applied to determine $k(T)$ in absorbing arcs : At low temperatures, radiation plays no role, consequently the second term of eq. (8) is negligible and $k$ may be determined directly from the first term which contains only known quantities. At higher temperature, radiation emission e will start to play a role. Having measured the total radiation $P_{r}$ leaving the column per unit length

$$
P_{\mathrm{r}}=2 \pi \int_{0}^{R} e r \mathrm{~d} r
$$

$e(T)$ may be determined by a numerical procedure similar to the one used to deduce $\sigma(T)$ from eq. (2). As long as only radiation emission without absorption plays a role in the energy balance, the function $k(T)$ determined from eq. (8) by using the known $\sigma(T)$ and $e(T)$ values will result in a unique function of temperature, regardless of the arc diameter used in the experiment. For high temperatures where $k(T)$ ceases to be a unique function for all radii used in the experiments, an extrapolation towards $R \rightarrow 0$ as described above may be used to determine temperature profiles and arc characteristics for arcs with vanishing diameter. By this procedure, it becomes possible to determine $k$ from eq. (8) even in cases of non-negligible radiation reabsorption. Figure 6 taken from reference [15] shows the resulting thermal conductivity of nitrogen at temperatures reaching $28000 \mathrm{~K}$. A comparison of this $k(T)$ (Fig. 7) with theoretical values [16] shows that the experiments lead to a substantially lower thermal conductivity in the temperature range up to $14000 \mathrm{~K}$ where the ionization peak occurs. At very high temperatures $(T>19000 \mathrm{~K})$

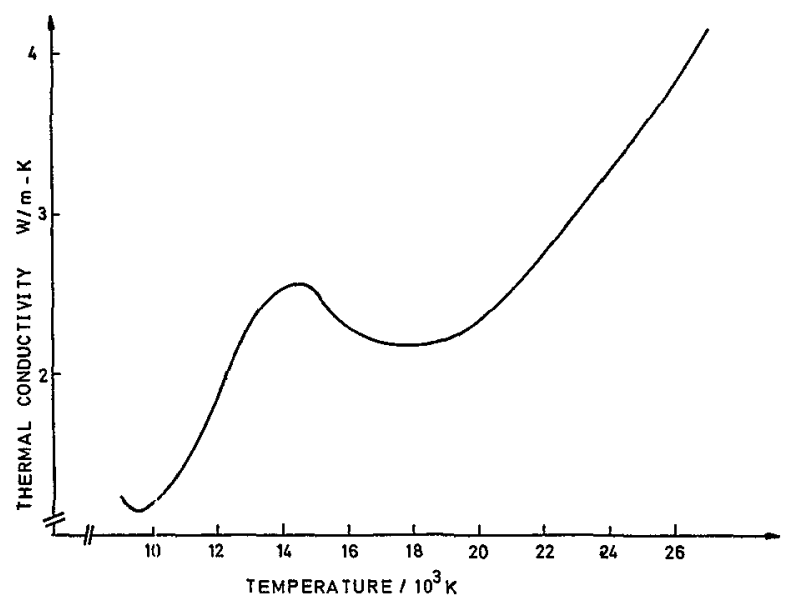

FIG. 6. - Nitrogen thermal conductivity [15].

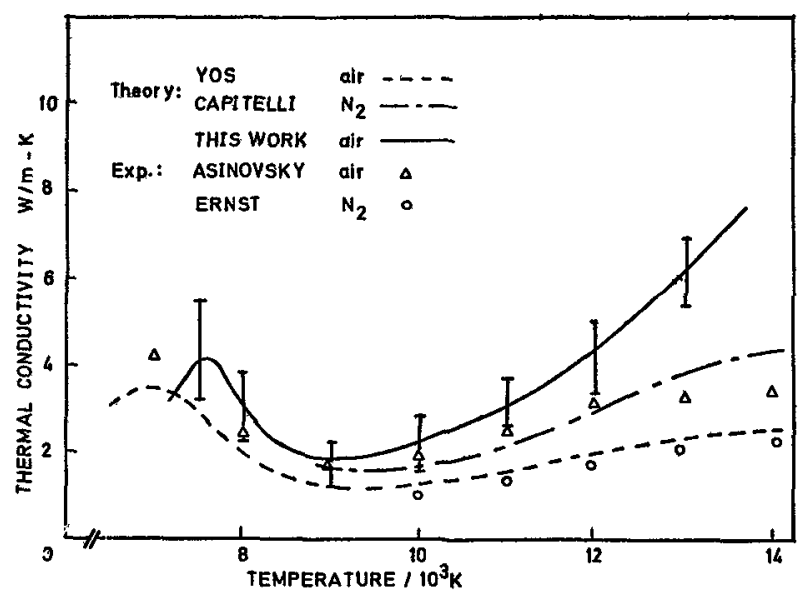

Fig. 7. - Air thermal conductivity [13].

the agreement of these data with theoretical values by Yos [17] is excellent.

In principle then, it is possible to determine the true thermal conductivity by the cascade arc technique even if radiation absorption plays a role. However, the number of experimental data required for an extrapolation $R \rightarrow 0$ is very large. Especially in the case of cascade arc experiments with air plasma where a substantial effort has to be directed towards the gas flow conditions in the test section (blanket gas in the electrode region), this experimental effort becomes excessive. For this reason, air thermal conductivity was determined from experiments with only one cascade diameter (Fig. 7) even at temperatures where absorption occurs within the plasma. As was to be expected, the resulting experimental data, containing a contribution related to absorption, exceed the theoretical values [17] as well as experimental data determined using a technique [14] similar to the technique used by Ernst for nitrogen plasma. 


\section{References}

[1] MarCKer, H. and Steinberger, S., Z. Angew. Phys. 23 (1967) 456.

[2] Boldt, G., Space Sci. Rev. 11 (1970) 728.

[3] OtT, W. R., Behringer, K. and Gieres, G., Appl. Opt. 14 (1975) 2121.

[4] LARENZ, R. W., Z. Phys. 129 (1951) 327.

[5] Einfeld, D., Stuck, D., Behringer, K. and Thoma, P., Z. Naturforsch. 31a (1976) 1131.

[6] Behringer, K. and Thoma, P., J. Quant. Spectrosc. Radiat. Transfer 16 (1976) 605 .

[7] Behringer, K. and ThOMA, P., J. Quant. Spectrosc. Radiat. Transfer 16 (1976) 671.

[8] GRIEM, H. R., Plasma Spectroscopy (McGraw-Hill, New York) 1964.

[9] Wiese, W. L., SMith, M. W. and Mrles, B. M., Atomic Transition Probabilities, Vol. II NSRDS-NBS 22 (1969).
[10] ScHLÜTER, D., Z. Astrophys. 56 (1962) 43.

[11] Bauder, U. H. and MAECKER, H., Proc. IEEE 59 (1971) 588.

[12] Kopainsky, J., Z. Phys. 248 (1971) 417.

[13] Devoto, R. S., Bauder, U. H., Shires, E. and Cailleteau, submitted for publication in Phys. Fluids.

[14] Asinovsky, E. I., Drokhanova, E. V., Kirillin, A. V., Lagaryhov, A. N., Templofiz. Vys. Temp. 5 (1967) 739.

[15] Ernst, K. A., Thesis Techn. Univ. Munich (1972).

[16] Capitelli, M., Devoto, R. S., Phys. Fluids 16 (1973) 1835.

[17] Yos, J. M., AVCO Report RAD-TM-63-7 (1963).

[18] Devoto, R. S., Phys. Fluids 19 (1976) 22.

[19] Asinovsky, E. I., Kirillin, A. V., Pakhomov, E. P., ShabaSHOv, V. E., Proc. IEEE 59 (1971) 592.

[20] SChreiber, P. W., Hunter, A. M., BENEDETto, K. R., AIAA Journal 11 (1973) 1815. 